\title{
Advantages of Surgical Implantation Impella 5.5 SmartAssist in the Management of Acute Post- Infarction Ventricular Septal Defect with Cardiogenic Shock
}

\section{Jeko Madjarov}

Sanger Heart and Vascular Institute

Michael G Katz ( $\square$ michael.katz1@mssm.edu )

Icahn School of Medicine at Mount Sinai https://orcid.org/0000-0003-4614-1485

Svetozar Madzharov

Sanger Heart and Vascular Institute

Shahood Fazal

Icahn School of Medicine at Mount Sinai

francis Robicsek

Sanger Heart and Vascular Institute

\section{Case report}

Keywords: Ventricular septal defect, Impella 5.5 SmartAssist, acute myocardial infarction, cardiogenic shock

Posted Date: August 5th, 2020

DOI: https://doi.org/10.21203/rs.3.rs-52596/v1

License: (c) (i) This work is licensed under a Creative Commons Attribution 4.0 International License.

Read Full License 


\section{Abstract}

ABSTRACT Background: Despite advances in surgical techniques and aggressive therapy of postinfarction ventricular septal defect (VSD) with cardiogenic shock, the overall morbidity and mortality is frustratingly high. The Impella 5.5 SmartAssist (Abiomed, Danvers, MA) is a surgically implanted temporary device, recently approved by the FDA for treatment of patients in cardiogenic shock, and may fill a technological gap for perioperative patients who require acute circulatory support. Case presentation: We report our initial experience with trans-aortic implantation of the Impella 5.5 SmartAssist for two patients with post myocardial infarction VSD in the setting of cardiogenic shock. First patient had a posterior VSD with a left to right shunt (Qp/Qs ratio of 3.3), right ventricle dysfunction, severe pulmonary arterial hypertension, and severe mitral valve regurgitation. Second patient was admitted for massive MI with large anterior VSD (Qp/Qs ratio of 2.8). Under cardiopulmonary bypass with cardioplegic arrest both patients underwent urgent VSD closure with trans-aortic implantation of the Impella. Minimal postoperative support was required. Patients were discharged on postoperative day 10 and 14 and remained well three months later. Follow-up echocardiogram showed no residual shunt. Conclusions: Early surgical implantation of Impella 5.5 SmartAssist can prevent multiorgan dysfunction and stabilize the patients in cardiogenic shock with post-myocardial infarction VSD.

\section{Background}

Ventricular septal defect (VSD) with cardiogenic shock remains a devastating complication following acute myocardial infarction (MI). Despite advances in surgical techniques and aggressive therapy including ventricular assist devices (VAD), the overall morbidity and mortality is frustratingly high. The timing of surgical intervention is critical. Moreover, no guidelines have been established for patient selection for early use of VADs in this setting. The Impella 5.5 with SmartAssist (Abiomed, Danvers, MA) is a surgically implanted temporary VAD recently approved by the FDA for treatment of patients in cardiogenic shock, and may fill a technology gap for perioperative patients who require acute circulatory support (Fig. 1A,B). This report summarizes our clinical experience with two patients with post-MI VSD in cardiogenic shock.

\section{Case presentation}

Patient 1. A 59-year-old man presented to our institution with acute inferior Ml and low blood pressure. Examination revealed a loud systolic murmur. Transthoracic echo demonstrated a large posterior VSD with a significant shunt (Qp/Qs ratio of 3.3), right ventricular dysfunction, severe pulmonary arterial hypertension, and akinesia of the inferior wall. Severe mitral valve (MV) regurgitation was observed. Coronary angiography displayed normal left main stem, 100\% RCA occlusion, and 40\% mid LAD stenosis. Cardiac index was 1.7 and a chest $\mathrm{X}$-ray revealed pulmonary edema. The patient was diagnosed with inferior $\mathrm{MI}$ and a large posterior VSD with cardiogenic shock $(<80 / 40 \mathrm{~mm} \mathrm{Hg})$, and underwent urgent VSD repair. 
Under cardiopulmonary bypass (CPB) with cardioplegic arrest, left ventriculotomy was made. A posterior VSD measuring $3.5 \times 4.5 \mathrm{~cm}$ in diameter was found (Fig. 1C). The septal myocardium surrounding the VSD was necrotic. Double patch technique to close VSD was used and several stitches were placed in the MV annulus, correcting the mitral insufficiency. A $10 \mathrm{~mm}$ graft was anastomosed to the distal ascending aorta. The Impella was introduced using manual manipulation and transesophageal echocardiography (TEE) guidance, prior to weaning patient from CPB. The device's $145^{\circ}$ angle assisted reposition the device away from the MV apparatus and VSD patch (Supplementary video).

Minimal postoperative support was required. Right ventricular (RV) load declined, as depicted by decreasing RV dimensions (Table 1). The device wean was guided by cardiac power output as Impella flow was reduced (Fig. 2). Impella removal did not require redo sternotomy. The graft was flushed, clamped, trimmed short, over sewn, and left in the subcutaneous space behind the sternal notch. The patient was discharged on postoperative day (POD) 10 and remained well three months later. Follow-up echocardiogram showed no residual shunt, and mild mitral regurgitation.

Table 1

Pre- and post-operative hemodynamic parameters

\begin{tabular}{|lll|}
\hline & $\begin{array}{l}\text { Preoperative hemodynamic } \\
\text { parameters }\end{array}$ & $\begin{array}{l}\text { Pre-discharge hemodynamic } \\
\text { parameters }\end{array}$ \\
\hline $\begin{array}{l}\text { Cardiac index, } \\
\mathrm{ml} / \mathrm{min} / \mathrm{m}^{2}\end{array}$ & 1.7 & 3.2 \\
\hline $\mathrm{Qp} / \mathrm{Os}$ & 3.3 & 1 \\
\hline $\mathrm{EF}, \%$ & 25 & 52 \\
\hline LV EDP, mmHg & 23 & 15 \\
\hline RV LTD, mm & 88 & 75 \\
\hline RV MCD, mm & 44 & 35 \\
\hline PAP systolic, mmHg & 72 & 35 \\
\hline PAWP, mmHg & 54 & 18 \\
\hline $\begin{array}{l}\text { EF: ejection fraction; LV EDP: left ventricular end-diastolic pressure; Qp/Qs: pulmonary to systemic } \\
\text { flow ratio; RV LTD: right ventricular longitudinal diameter; RV MCD: right ventricular mid-cavity } \\
\text { diameter; PAP: pulmonary artery pressure; PAWP: pulmonary artery wedge pressure }\end{array}$ \\
\hline
\end{tabular}

Patient 2. A 60-year-old male was admitted for massive anterior MI with CS. Work-up showed proximal LAD occlusion, a large anterior VSD with a left-right shunt (Qp/Qs ratio of 2.8) and ejection fraction of $25 \%$. Despite intra-aortic balloon counter pulsation and inotropic support, the patient developed shock with cardiac index of 1.8 and serum lactate of $6 \mathrm{mmol} / \mathrm{L}$. In the operating room, a ventriculotomy was performed. The VSD $(2.5 \times 2.8 \mathrm{~cm}$; Fig. 1D) was repaired with a Bovine pericardial patch and CABGx1 was carried out. The Impella 5.5 was implanted using the technique described above (Fig. 1E). The SmartAssist technology recognized "ventriculization" of the device upon arrival in the ICU and 
repositioning resulted in recovery of hemodynamics. The patient was discharged with a life vest on POD 14 , remaining asymptomatic and well on virtual 3-month follow-up visits.

\section{Discussion}

Cardiogenic shock on presentation and preoperative deterioration of hemodynamics are strong predictors of mortality. ${ }^{1,2}$ Although AHA guidelines recommend early surgical repair regardless of hemodynamic status, the timing of surgery and use of VADs in the setting of post-MI VSD with cardiogenic shock remains controversial and should be individualized. ${ }^{3}$ The main challenge in treating patients with post-MI VSD is whether to correct the defect immediately or to delay surgical closure and allow time for tissue and organ recovery. ${ }^{2}$ For our patients in cardiogenic shock, we opted for early surgical repair of VSD with intraoperative Impella placement to allow immediate LV decompression with continuous drains of LV, thus maintaining flow from LV to the aorta, preventing worsening of cardiac performance and organ malperfusion, while resolving the pulmonary to systemic shunt. ${ }^{4}$ We believe that this technique protects the integrity of the VSD patch, providing additional time for septal tissue maturation, and it gives surgeons the opportunity to perform concomitant interventions (i.e, CABG, valve repair/replacement etc.).

In the recent years, the peripheral arterial approach in implanting catheter-based VADs has become the predominant technique ${ }^{5}$. Due to the urgency in cardiogenic shock in our patients, we placed the device through a graft sewn to the distal ascending aorta during CPB; a strategy more time sensitive than an axillary or femoral approach. Moreover, trans-axillary Impella placement in such cases is beneficial for LV venting and continued support allowing for early bed mobilization of the patients compared to femoral access.

Extracorporeal membrane oxygenation (ECMO) is commonly used in such patients; however, ECMO has been shown to compromise LV contractile function with increased LV end-diastolic pressure, LV afterload, impaired myocardial blood flow and often requires additional intervention such as LV venting. In cases of advanced cardiogenic shock with severe RV dysfunction and multi-organ failure, we have previously utilized ECMO with Impella support. The Impella 5.5 SmartAssist is less invasive and provides more practical and effective postoperative treatment and mechanical support in LV dysfunction with myocardial stunning than ECMO.

With a forward flow to the general circulation of up to $6.2 \mathrm{~L} / \mathrm{min}$ and optical sensor technology, the Impella 5.5 SmartAssist is best able to fully decompress the heart of all currently available mechanical circulatory support devices. This is particularly important in patients with large body habitus or in cardiogenic shock with a vasodilatory component, which may be the result of suboptimal LV decompression. Other advantages of the Impella 5.5 include device removal and repositioning at bedside with SmartAssist technology; furthermore, we observed an improvement in RV function with resolution of pulmonary edema and mitral insufficiency. These advantages might lower the decision threshold for the initiation of mechanical circulatory support to restore the hemodynamics, preventing the aggravation of 
organ failure and potentially leading to improved outcomes in patients with post-infarction VSD in cardiogenic shock.

\section{Conclusions}

To our knowledge, this is the first report of surgical implantation of the Impella 5.5 SmartAssist for patients with post-infarct VSD in cardiogenic shock. Our experience suggests the feasibility of using the Impella 5.5 SmartAssist to stabilize the patient, lowering pulmonary arterial and wedge pressure, reduce mitral valve incompetence and support the stunned ventricle with improvement of cardiac index after early surgical VSD closure, allowing heart recovery while maintaining distal organ perfusion. Our findings concerning the unique utility of the Impella 5.5 in the setting of cardiogenic shock merit broader exploration.

\section{Abbreviations}

VSD

Ventricular septal defect; Post-Ml:post-myocardial infarction; VAD:ventricular assist device; CPB:cardiopulmonary bypass; MV:mitral valve; LV:left ventricle; POD:postoperative day; LAD:left anterior descending artery; ECMO:Extracorporeal membrane oxygenation

\section{Declarations}

\section{Acknowledgements}

We would like to acknowledge Dana Bentley, MWC, for editorial assistance and Abiomed Inc for providing illustrations.

\section{Authors' contributions}

$\mathrm{JM}$ performed the surgical procedure. JM and FR were involved in the patient's care. MK collected the clinical data. JM, MK, SM, and SF prepared the manuscript. All authors revised the manuscript and approved the final manuscript.

\section{Funding}

This work was partially supported by Heineman Foundation Grant for research and education.

Auxiliary medical writing assistance was funded by the device manufacturer and in accordance with Good Publication Practice.

\section{Availability of data and materials}


Not applicable.

\section{Ethics approval and consent to participate}

Not applicable.

\section{Consent for publication}

The patients signed informed consent.

\section{Competing interests}

None declared

\section{References}

1. Jentzer JC, van Diepen S, Barsness GW, Henry TD, Menon V, Rihal CS, et al. Cardiogenic Shock Classification to Predict Mortality in the Cardiac Intensive Care Unit. J Am Coll Cardiol. 2019;74(17):2117-28.

2. Deja MA, Szostek J, Widenka K, Szafron B, Spyt TJ, Hickey MS, et al. Post infarction ventricular septal defect - can we do better? Eur J Cardiothorac Surg. 2000;18(2):194-201.

3. O'Gara PT, Kushner FG, Ascheim DD, Casey DE, Jr., Chung MK, de Lemos JA, et al. 2013 ACCF/AHA guideline for the management of ST-elevation myocardial infarction: a report of the American College of Cardiology Foundation/American Heart Association Task Force on Practice Guidelines. Circulation. 2013;127(4):e362-425.

4. Gregoric ID, Bieniarz MC, Arora H, Frazier OH, Kar B, Loyalka P. Percutaneous ventricular assist device support in a patient with a postinfarction ventricular septal defect. Tex Heart Inst J. 2008;35(1):46-9.

5. Tepper S, Masood MF, Patel K, Garcia MB, Pisani M, Ewald GA, et al. Left ventricular unloading by Impella device versus surgical vent during extracorporeal life support. Ann Thorac Surg. 2017;104:861-7.

\section{Figures}




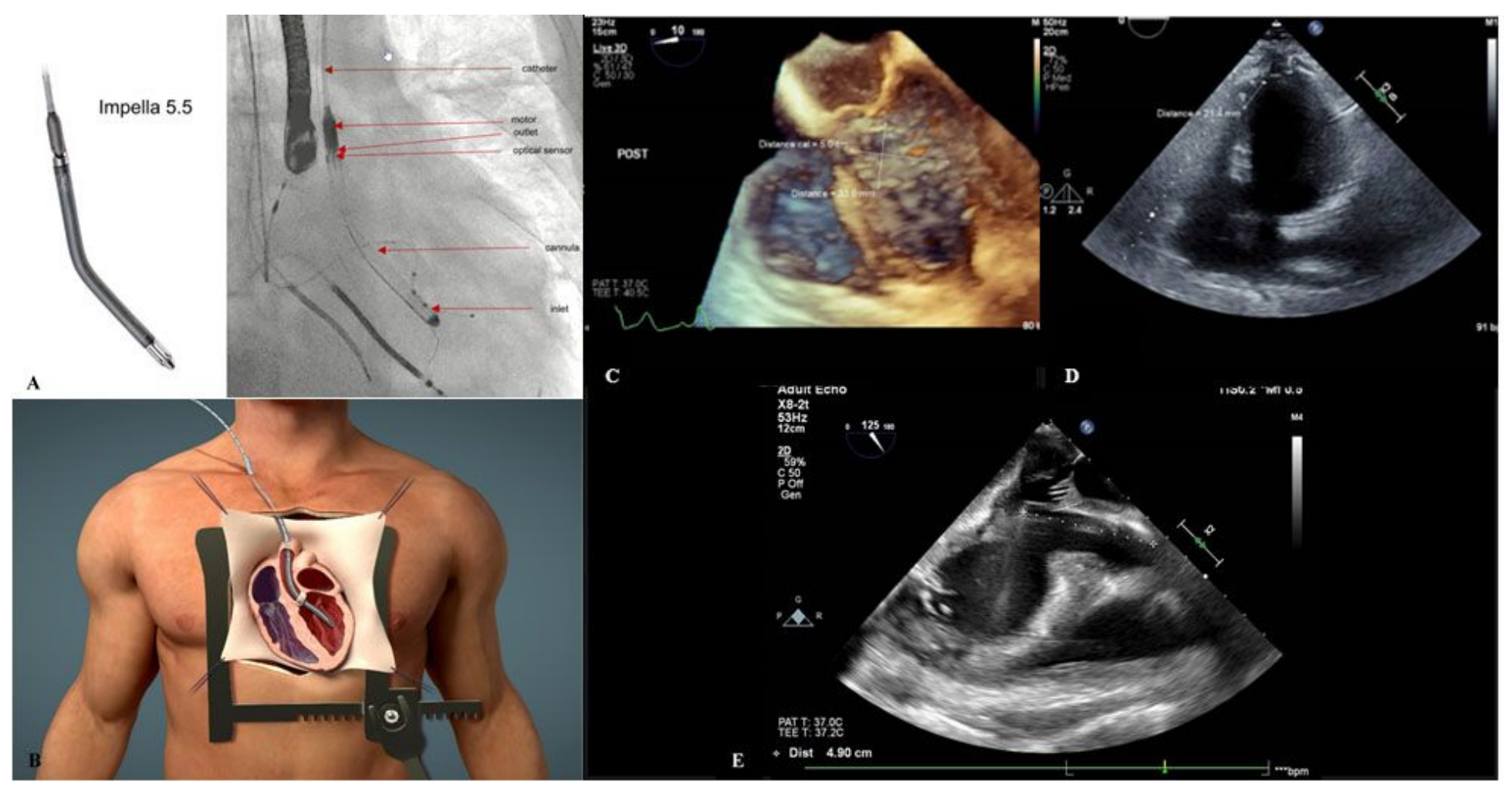

Figure 1

A, The Impella 5.5 SmartAssist in situ. B, Trans-aortic Impella placement during VSD repair. C, Transesophageal echocardiography (TEE) image of the anterior VSD. D, TEE image of the posterior VSD. $\mathrm{E}, \mathrm{TEE}$ image of Impella placement in Patient 2 (anterior VSD). 
Flow

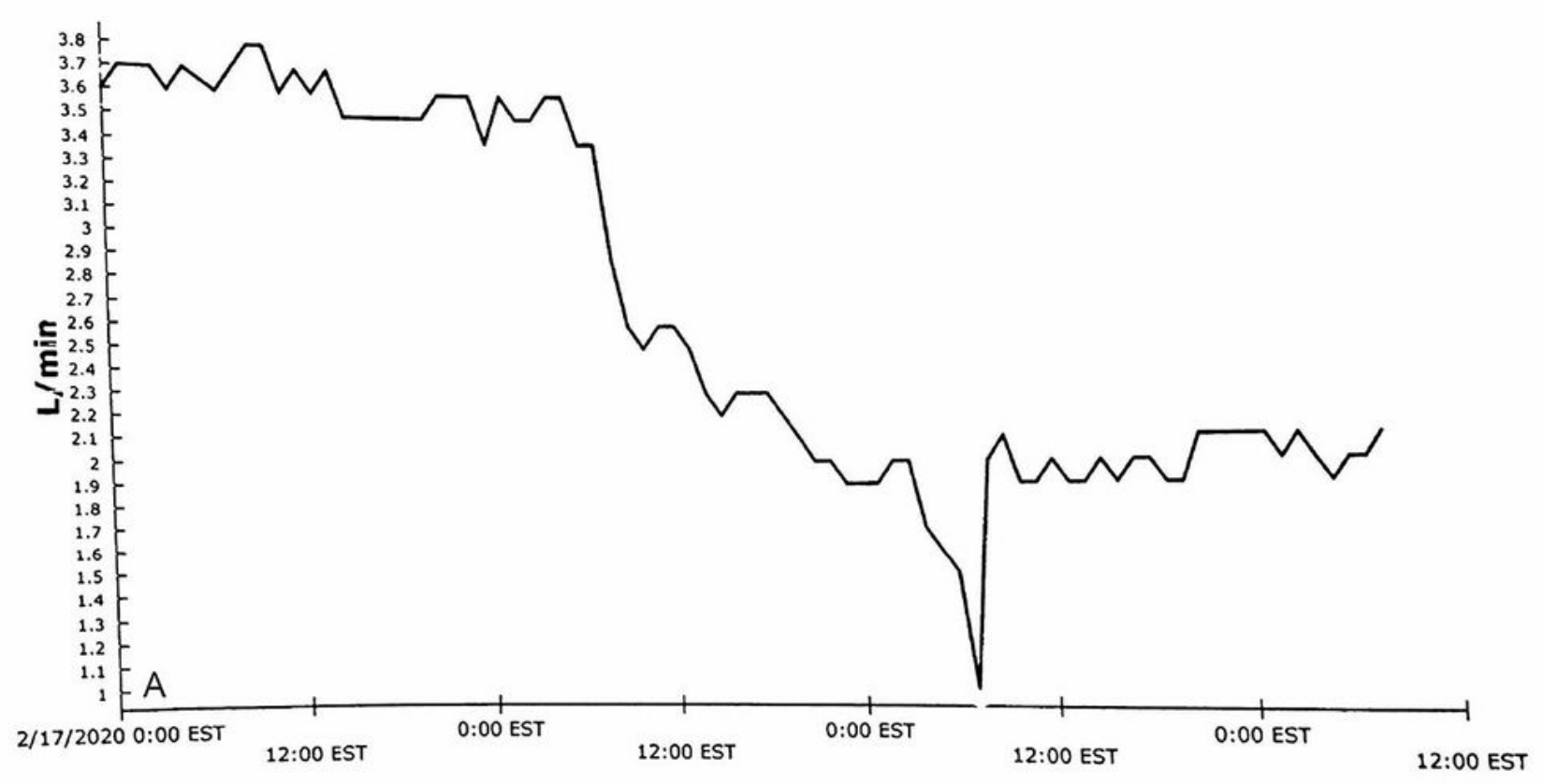

Flow

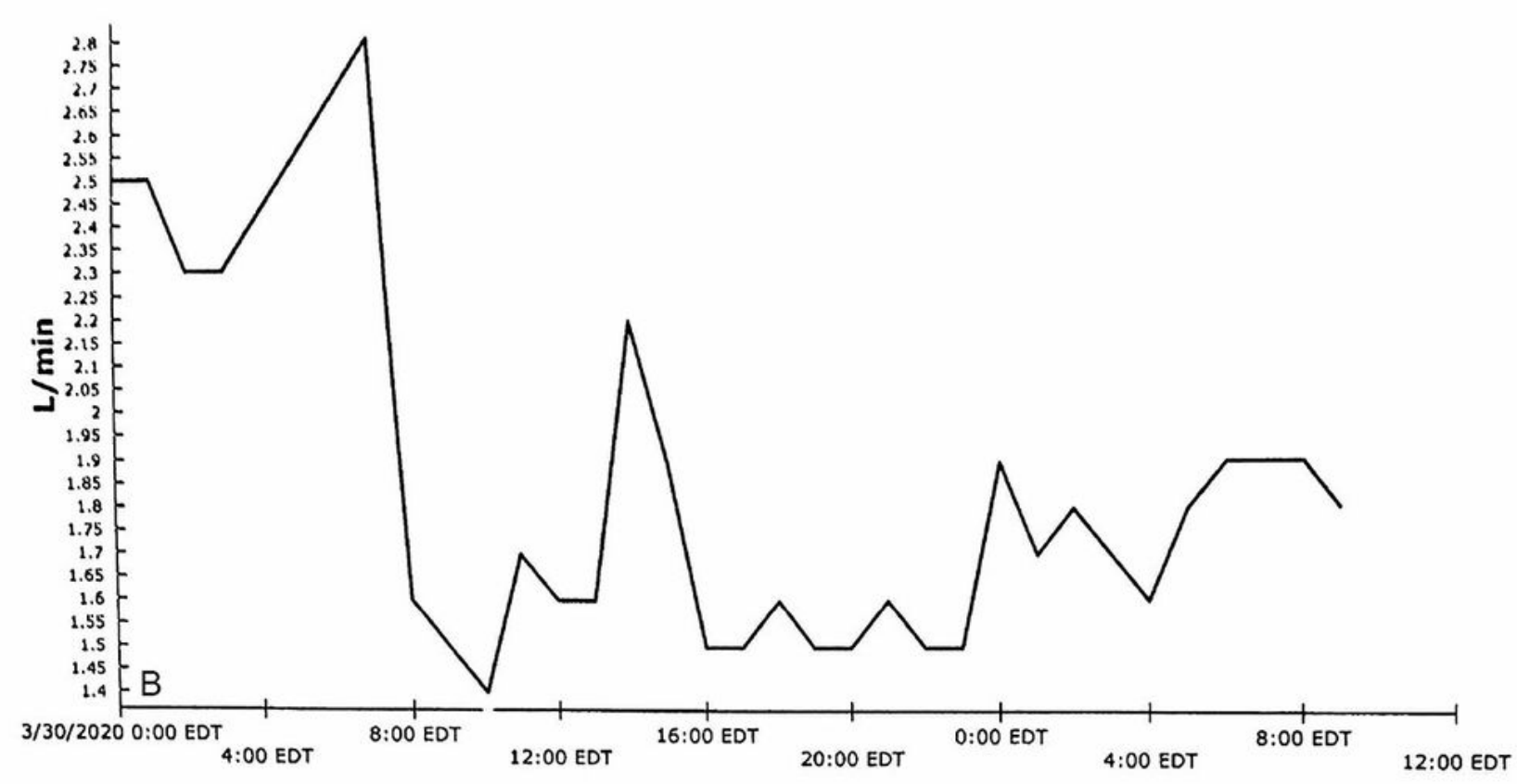

\section{Figure 2}

The SmartAssist technology provided parameters to guide weaning. Flow was decreased by one power setting every 12-24 hours for 12 consecutive hours while maintaining cardiac power output $>0.6$ with the patient ambulating. A, Patient 1 had Impella removal on POD 6 (ambulation on POD 2). B, Patient 2 had Impella removal on POD 10 (ambulation on POD 3). 


\section{Supplementary Files}

This is a list of supplementary files associated with this preprint. Click to download.

- CAREchecklistEnglish2013.pdf

- Video1 copy.mp4 